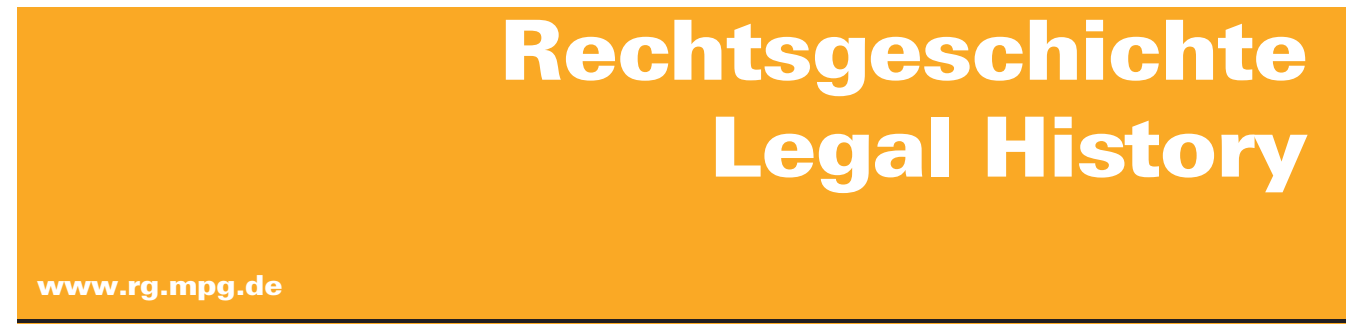

http://www.rg-rechtsgeschichte.de/rg23

$\mathrm{Rg} 232015 \quad 331-336$

Zitiervorschlag: Rechtsgeschichte - Legal History Rg 23 (2015)

http://dx.doi.org/10.12946/rg23/331-336

\title{
Leila Scheuch
}

\section{Unrecht der Ehe}

Zur Diskussion und Umsetzung der Rechte verheirateter Frauen im 19. und frühen 20. Jahrhundert 
que Oxford University Press ha logrado nuevamente su objetivo de publicar un libro con uno solamente.

Observo, impecabilidad editorial aparte que, cuando adopta la primera persona, el autor usa el plural mayestático. Ya en la lengua castellana esto suena bastante raro en nuestra parte del mundo, pues se suele reservar a majestades y sumos pon- tífices; pero en el inglés en el cual el libro fue publicado se lo siente (al pronombre «we») todavía menos convencional y, más aún, paradójico, en una obra destinada a erigirse en un bastión del igualitarismo constitucional latinoamericano.

\section{Laila Scheuch}

\section{Unrecht der Ehe}

Zur Diskussion und Umsetzung der Rechte verheirateter Frauen

im 19. und frühen 20. Jahrhundert*

>Ungerechtigkeit< und >Ungleichheit ‘ waren zwei Schlüsselworte der nationalen wie internationalen Frauenbewegung im Hinblick auf die rechtliche Situation von Frauen zu Beginn des 20. Jahrhunderts. ${ }^{\mathbf{1}}$ Ein von Stephan Meder und Christoph-Eric Mecke 2013 herausgegebener, ausgesprochen lesenswerter und sehr übersichtlich konzipierter Sammelband rückt nun ein bis dato in der Forschung eher vernachlässigtes Thema und Tätigkeitsfeld der Frauenbewegungen des 19. und frühen 20. Jahrhunderts in den Mittelpunkt: das Familienrecht. Mit diesem Interesse stehen die beiden Herausgeber keinesfalls alleine da. Zwei weitere im selben Jahr erschienene Sammelbände, die ebenfalls sehr lohnende Lektüre bieten, lenken ihre Aufmerksamkeit auf die Bedeutung des Familienrechts für Frauen. Die in Married Women and the Law zusammengestellten Aufsätze gehen der normativen und praktischen Bedeutung des für verheiratete Frauen in England und Nordamerika zentralen Rechtskonzepts der coverture vom Mittelalter bis zur »Flut von Reformen im späten 19. Jahrhundert ${ }^{2}{ }^{2}$ (4) auf den Grund. Unter coverture verstanden common law-Juristen die Idee, dass Frauen mit der Heirat ihre eigene rechtliche Identität verlören und von der ihres Mannes sbedeckt (covered) würden. Daraus folgten hauptsächlich personen- und eigentumsrechtliche Einschränkungen für Ehefrauen. Sie verloren u. a. das Recht, Eigentum zu besitzen oder zu verwalten, Verträge einzugehen oder ohne ihren Ehemann eine Klage anzustrengen. Die Artikel in Gender Difference in European Legal Cultures weisen demgegenüber eine breitere thematische Vielfalt auf, was in der Frage-

\footnotetext{
* Karin Gottschalk (Hg.), Gender Difference in European Legal Cultures. Historical Perspectives, Stuttgart: Steiner 2013, 261 S., ISBN 978-3-515-09409-2 Stephan Meder, Christoph-Eric Mecke (Hg.), Family Law in Early Women's Rights Debates. Western Europe and the United States in the $19^{\text {th }}$ and early $20^{\text {th }}$ centuries, Köln: Böhlau 2013, 410 S. ISBN 978-3-412-21052-6 Tim Stretton, Krista J. Kesselring (Hg.), Married Women and the Law.
}

Coverture in England and the Common Law World, Montreal: McGill Queens University Press 2013, 281 S., ISBN 978-0-7735-4292-1, 978-0-7735-4297-6, 978-0-7735-9013-7, 978-0-7735-9014-4

1 Vgl. International Council of Women (Hg.) (1912), Women's Position in the Laws of the Nations, Karlsruhe, bes. S. VI f. Gerhard, Family Law 30. RöweKAMP, Family Law 212.

2 Diese und alle folgenden Übersetzungen aus dem Englischen sind von der Autorin. 
stellung des Bandes begründet liegt. Im Fokus stehen hier »die rechtlichen Normen, die sich explizit auf Männer und Frauen beziehen« (11). Es wird danach gefragt, "was die Funktion von Geschlecht in der Schaffung von Recht; und umgekehrt, was die Funktion von Recht in der Schaffung von Geschlecht" sei (11). Dabei geraten der europäische Subkontinent mit Ausnahme Russlands und der weitere Mittelmeerraum vom Mittelalter bis zur Neuzeit in den Blick. Die Fallbeispiele fallen zum Teil sehr unterschiedlich aus (u.a. jüdische Juristinnen, Abtreibung, Inter- und Transsexualität), ein Schwerpunkt zeigt sich aber im Bereich des Güterrechts.

Im Folgenden werden ausgehend von Family Law jene Beiträge der drei Publikationen näher beleuchtet, die sich mit der familienrechtlichen Situation verheirateter Frauen im 19. und frühen 20. Jahrhundert in den Rechtskreisen auseinandersetzen, die vorrangig in der Forschung behandelt werden bzw. die in Meder/Mecke fehlen. Eine Ergänzung der Betrachtung von Family Law um Gender Difference und Married Women bietet sich aufgrund der frappierenden Gemeinsamkeiten der Bände - trotz ihrer unterschiedlichen thematischen und zeitlichen Breite - an. Dies beginnt bei ihrem gleichen Entstehungshintergrund im Gefolge von Konferenzen und endet bei allen eigenen theoretisch-methodischen Präferenzen. Zunächst einmal ist nicht nur Married Women, sondern sind auch die von deutschsprachigen Herausgebern und in verstärkt deutschen Forschungskontexten entstandenen anderen beiden Bücher mit einer einzigen Ausnahme in Englisch verfasst. ${ }^{3}$ Mit dem Gebrauch der lingua franca tragen die Herausgeber/-innen ihrer internationalen Autorenschaft Rechnung und erhöhen die Les- und Sichtbarkeit über nationale Sprachbarrieren hinaus. Tim Stretton und Krista J. Kesselring bemühen Judith Bennetts Idee des patriarchal equilibrium, also von »Wandel ohne Umwandlung« (264), bei ihrer zentralen Frage nach den Gründen für die »erstaunliche Beständigkeit« (11) der »Regeln und Effekte von coverture hinsichtlich einer ganzen Reihe von rechtlichen, sozialen, ökonomi- schen, kulturellen und religiösen Veränderungen « (5). Die Antworten sind dabei gezielt interdisziplinär ausgerichtet - die Beiträgerinnen forschen u. a. literatur- und wirtschaftswissenschaftlich. In Family Law übernimmt Ute Gerhard diesen Teil. Sie fordert eine interdisziplinäre Vorgehensweise bei der Erforschung der Frauenrechtsbewegung und belegt die Fruchtbarkeit dieses Ansatzes mit einer soziologischen Interpretation der modernen Frauenbewegung als einer »sozialen Bewegung par excellence $(29$ k. i. O.). Karin Gottschalk problematisiert die Wechselbeziehungen von Geschlecht und Recht bei der Konstruktion sozialer und politischer Ordnung und arbeitet somit, wie viele der Autoren/-innen von Gender Difference, ebenfalls interdisziplinär mit feministischen Konzepten an der Schnittstelle von Rechts-, Geschichts- und Politikwissenschaft.

Neben der Interdisziplinarität als Leitlinie für die Bearbeitung der Thematik heben die Herausgeber/-innen und Ute Gerhard die große Nützlichkeit komparativer Herangehensweisen hervor. Karin Gottschalk verdeutlicht dies am eindringlichsten, wenn sie ausführt, dass Vergleichen nicht nur Wissen schaffe, sondern auch den Blick für Verflechtungs- und Translationsvorgänge öffne, in denen sich kulturelle Identität - in der wiederum Geschlecht als Strukturkategorie eine jeweils spezifische Rolle spielen kann - herausbilde. So belege der diachrone und synchrone Vergleich, den die Artikel des Buches ermöglichen, „wie immens wichtig die Art, in der das Recht in verschiedenen Gesellschaften organisiert ist, für die Geschlechterdifferenz" sei (31). Zudem wird der Verlust des Eurozentrismus als erwünschtes Resultat komparativer Forschung genannt. Auf diese Aspekte spielt auch Gerhard an. Hervorzuheben ist in diesem Zusammenhang besonders ihr Hinweis, dass die Frauenbewegung selbst bereits seit ihren Anfängen in der Mitte des 19. Jahrhunderts transnational war. Der Vergleich zwischen den Frauenbewegungen einzelner Länder sowie der internationalen Organisationen, so Meder und Mecke in der Einführung, decke auf, dass sich Etiketten wie sprogressiv oder gar >radikak keinesfalls einfach
3 Gender Difference: International Research Network Gender Difference in the History of European Legal Cultures (mit starker deutscher Beteiligung u. a. an der Gründung); Family Law: DFG-Projekt »Internationale
Reformforderungen zum Familienrecht und Rechtskämpfe des Frauenweltbundes 1830-1914« an der Universität Hannover. Ausnahme: GEIGER in Gender Difference, 175-186. 
auf die Vertreter/-innen der Forderungen nach dem Frauenwahlrecht kleben ließen, respektive sgemäBigt< oder >konservativ « auf diejenigen, die Verbesserungen der familienrechtlichen Situation von Frauen verlangten. Stretton und Kesselring bleiben hinsichtlich vergleichender Methodik etwas >untertheorisiert . Ihnen dient der Vergleich als Mittel zum Zweck des »tieferen Verständnisses der Effekte des Rechts auf das Leben verheirateter Frauen« (266). Insgesamt bewegen sich alle drei Bände mit der interdisziplinären, komparativen und internationalen Ausrichtung auf dem neuesten Stand der Geschichtswissenschaft.

Family Law ist im Gegensatz zum chronologischen Aufbau der anderen beiden Sammelbände nach internationaler und nationaler Handlungsebene der Frauenrechtsbewegungen strukturiert, wobei die »Internationale Perspektive: Transnationale Frauenrechtsdebatten « den Auftakt macht. Darauf folgen Frankreich, Belgien, Italien - drei Länder in der Rechtstradition des französischen Code civil, dann die Länder in common law-Tradition England, Wales und die USA und schließlich Skandinavien als ein weiterer Rechtsraum. Den Abschluss bildet eine "Historische Perspektive: Antikes Römisches Recht«. Ergänzt werden die Artikel durch Transkriptionen der Diskussionen auf der zugrundeliegenden Konferenz. Arbeiten zum deutschsprachigen Rechtsraum fehlen - eine Lücke, die durch Beiträge in Gender Difference geschlossen werden kann. Das Hinzuziehen von Married Women deckt einen Schwerpunkt der Forschung im angloamerikanischen Raum auf.

Die frühe internationale Vernetzung von Frauenrechtlern/-innen belegt eindrucksvoll das Gründungsdatum des International Council of Women (ICW), 1888, der somit vor den nationalen Organisationen gegründet wurde. Seine fortschreitende Institutionalisierung in den folgenden drei Jahrzehnten charakterisiert Anja Schüler (Family Law) als typisch für die Herausbildung internationaler Organisationen in der zweiten Hälfte des 19. Jahrhunderts. Neben Bildung, sozialen Fragen, Wahlrecht und Erwerbsarbeit setzte sich der ICW ausführlich mit der rechtlichen Lage von Frauen auseinander, wobei diese Themen vielfältig miteinander verknüpft waren. Als Ergebnis des ICW Kongresses in Toronto 1909 erstellten die nationalen Mitgliedsorganisationen Berichte zu ungleichen Gesetzen für Männer und Frauen des jeweiligen Landes, die 1912 zusammengefasst vom ICW als Women's Position in the Laws of the Nations veröffentlicht wurden. Women's Position zeige beispielhaft, wie »internationale Organisationen im entstehenden >global age arbeiteten: Als Teil einer sich ausdehnenden internationalen Zivilgesellschaft, sorgten sie für einen Informationsfluss über Grenzen hinweg für ihre Mitglieder und die breite Öffentlichkeit in den Mitgliedsnationen« (66). Diese Strategie des ICW sei durchaus erfolgreich gewesen, denn der internationale Vergleich habe den nationalen Frauenrechtsorganisationen beim Lobbying für ihre Ziele geholfen. Auch Karen Offen (Family Law) beurteilt die Arbeit des ICW auf lange Sicht als grundsätzlich gewinnbringend, in diesem Fall für die Forderungen der französischen Frauenbewegung, die seit ihren Anfängen in der ersten Hälfte des 19. Jahrhunderts u. a. auf eine Verbesserung des Rechtsstatus von Ehefrauen, welche vielfältigen rechtlichen Diskriminierungen ausgesetzt waren, drangen. Über Aktivistinnen wie die Anwältin Maria Vérone oder Mme d'Abbadie d'Arrast waren die französischen Feministen/-innen um die Jahrhundertwende und danach in leitenden Positionen an der internationalen Frauenrechtsbewegung beteiligt.

Trotz der gemeinsamen Rechtstradition - der belgische Code civil trat 1804 in Kraft und folgte dem französischen Vorbild - traten Frauenrechtler/-innen in Belgien im Vergleich zu Frankreich und den weiteren Nachbarländern erst sehr spät in öffentliche Erscheinung, nämlich gegen Ende des 19. Jahrhunderts (Catherine Jacques, Family Law). Obwohl der belgische Feminismus sich auf die rechtliche Unterlegenheit der Frau gegenüber ihrem Mann in der Ehe mit dem daraus folgenden »kompletten rechtlichen Unvermögen« konzentrierte (113), war die Bewegung spätestens ab 1920 stark gespalten und bietet ein Beispiel dafür, wie religiöse und politische Ansichten feministische Forderungen beeinflussten und zur Spaltung der Bewegung beitragen konnten. Die sogenannten >christlichen Feministen/-innen in Belgien, die dem katholischen Glauben angehörten, wollten die Hierarchie in der Ehe nicht abschaffen, sondern sie weniger harsch für Frauen gestalten, damit diese besser ihren familiären Aufgaben nachkommen könnten. Im Gegensatz dazu traten die segalitären Feministen/-innen`, meist vor dem Hintergrund einer liberal-demokratischen oder sozialistischen Ausrichtung, für eine Abschaffung des Artikels 213, der die Frau zum Gehorsam gegenüber ihrem Mann verpflichtete, ein. Die italienische Frauenrechtsbewegung entwickelte sich seit der 
nationalen Einigung Italiens $1859 / 60$ gleichfalls vor dem Hintergrund des französischen Code civil, von dem im Familienrecht einige der patriarchalen Prinzipien in den neuen Codice civile, der Anfang Januar 1866 in Kraft trat, übernommen wurden (Dickmann, Family Law). Anna Maria Mozzoni, die »Gründerin« (150) der italienischen Bewegung, ist eine weitere transnationale feministische Akteurin, indem sie sich auch auf der internationalen Ebene einbrachte. Im Zentrum ihrer familienrechtlichen Forderungen in Italien stand ähnlich wie in Frankreich die Schaffung rechtlicher Gleichheit zwischen verheirateten und unverheirateten Frauen, welche wesentlich unabhängiger handeln konnten als die erstgenannten. Ähnlich den belgischen segalitären Feministen/-innen ‘ war Mozzonis Agenda von ihrer sozialistischen Überzeugung beeinflusst. Sozialer und demokratischer Fortschritt könne sich nur in einer Gesellschaft aus gleichberechtigten Frauen und Männern erfüllen.

Die Forschung zur rechtlichen Benachteiligung von Ehefrauen im common law-Land England treibt besonders stark die Frage nach dem Einfluss der Frauenbewegungen auf die Familienrechtsreformen der zweiten Hälfte des 19. Jahrhunderts um, wie den Divorce and Matrimonial Causes Act 1857, den Custody of Infants Act 1873 und den Married Women's Property Act 1882. Beide Autorinnen, die sich mit der Frage beschäftigen, betonen den signifikanten Einfluss anderer Faktoren. Rebecca Probert (Family Law), deren Artikel eine sehr differenzierte und kenntnisreiche Antwort gibt, argumentiert, dass die Reformen zwar oftmals im Einklang mit Forderungen von Frauenrechtsgruppen standen, letztlich aber eine ganze Reihe von Umständen zu den Verabschiedungen beitrugen. Dazu gehörten das Handeln einer individuellen Person, nämlich Caroline Norton, die Unterstützung durch reformorientierte Organisationen wie die Law Amendment Society und populäre Schriften wie die John Stuart Mills zur »Hörigkeit der Frau« (1869), die Art des parlamentarischen Diskurses, der anstatt auf Gleichheit auf Schutz als Argument rekurrierte und auf vorherige Reformen als Vorbild für weitere verwies, sowie ökonomische Veränderungen. Trotzdem plädiert Probert dafür, die Wirkung der englischen Frauenrechtsbewegung nicht unterzubewerten, denn für diese hätte zunächst einmal die Öffnung des öffentlichen Raums für Frauen die essenziellere Bedeutung besessen als Familienrechtsreformen. Zusätzlich seien die Reformen nach dem Ersten Weltkrieg ohne die frü- here Agitation nur schwer durchführbar gewesen. Mary Beth Combs (Married Women) konzentriert sich auf die Verabschiedung des Married Women's Property Act von 1870. Als entscheidenden Grund für die Verabschiedung des Act 1870, obwohl eine Reform der Besitzrechte von Ehefrauen bereits in den 1850er Jahren in der Öffentlichkeit und im Parlament debattiert wurde, sieht sie die Lobbyarbeit von Kaufleuten, die "sich über eine Steigerung in Betrugsfällen, die Sondervermögen von verheirateten Frauen beinhalteten, beschwerten « (218). Solche Sondervermögen waren im Rahmen des common law-Prinzip der coverture gar nicht zulässig, im Rahmen von equity-Regelungen jedoch schon, so dass Ehepaare die Widersprüche zwischen equity und common law zu ihren Gunsten nutzen konnten. Die ökonomische Perspektive in Verbindung mit der Uneinheitlichkeit der Rechtsnormen fügt dem bereits komplexen Gefüge an Gründen, das von Probert dargestellt wurde, eine weitere Komponente hinzu. Für sich allein betrachtet, ist diese Argumentation jedoch reduktionistisch.

Danaya C. Wright (Married Women) erweitert diese strukturengeleiteten Perspektiven und fragt nach der Handlungsfähigkeit von Ehefrauen in England und den USA im 19. Jahrhundert im Rahmen der sie betreffenden rechtlichen Restriktionen. Mittels eines mikrohistorisch-biographischen Vorgehens kann sie vielfältige Methoden des »Widerstands« (242) sowohl öffentlicher als auch privater, formeller und informeller Natur, von Frauen beider Länder gegen die coverture belegen. Die Widerstandsakte bezogen sich zumeist auf Fragen der Kontrolle von Besitz - Wright weist nach, dass fast alle der Frauen ein deutliches Bewusstsein für Eigentumsverhältnisse besaßen sowie des Zugangs zu Kindern und des Sorgerechts für diese. Bonnie S. Anderson (Family Law) wählt gleichermaßen einen biographischen Zugang. Am Beispiel von Ernestine Rose, einer führenden Rednerin in der frühen amerikanischen Frauenrechtsbewegung, die sich neben Elizabeth Cady Stanton um 1860 für das Scheidungsrecht engagierte, zeigt sie zum einen die große Bedeutung religiöser bzw. säkularer Normen für die Forderungen der Frauenrechtler/-innen, zum anderen wird hier erneut die Spätwirkung als die eigentliche Leistung der Frauenbewegung des 19. Jahrhunderts gewürdigt. Im Gegensatz zu anderen Scheidungsbefürworterinnen, habe Rose eine säkulare, "wesentlich modernere Begründung « für das Scheidungsrecht for- 
muliert als beispielsweise Stanton, die "Geschlechterstereotypen, Sentimentalität, und Religiosität sowie feministische Prinzipien« angeführt habe (238). Damit sei sie eine der geistigen Urheberinnen gegenwärtiger Konzeptionen des Scheidungsrechts ohne Verschuldungsprinzip. Grundsätzlich jedoch war Scheidung ein äußerst kontroverses Thema innerhalb der Bewegung, nicht zuletzt weil Religion eine "mächtige Kraft« bis in das 20. Jahrhundert hinein blieb (247).

Ein weniger zwiespältiges Thema war das Sorgerecht für Kinder, ein Bereich, in dem die amerikanische Frauenrechtsbewegung relativ beständig Reformen forderte, da die bestehenden Sorgerechtsregelungen als »Beweis - häufig führender Beweis - für die Ungerechtigkeit bestehender Gesetze« gesehen wurden (Röwekamp, Family Law 212). Zwischen 1890 und 1920 habe es eine erste "Sorgerechtsreformwelle" gegeben, eine zweite zwischen 1920 und 1930, die jeweils zu einer Ausweitung der Ansprüche von Frauen auf das Sorgerecht über ihre Kinder in einzelnen Bundesstaaten führten. Dennoch existierten bis zu den 1960er bis 80er Jahren in einigen Staaten weiterhin (sorge-)rechtliche Ungleichheiten zwischen den Geschlechtern, so dass Röwekamp ebenfalls die Frage nach dem Erfolg der Frauenbewegung beschäftigt. Während es ihr zuzurechnen sei, das Thema um 1850 in den öffentlichen Diskurs gebracht und dort gehalten zu haben, führt sie darüber hinaus eine Reihe struktureller Änderungen an, die die Reformen im Sinne Proberts erleichterten. Dazu zählen der Wandel kultureller Vorstellungen, die sich in der Schaffung von neuen juristischen Termini wie $>$ Kindeswohl manifestierten oder darin, dass der Staat zunehmend als kompetent für die Regelung von Familienangelegenheiten angesehen wurde, die Unterstützung männlicher Befürworter, aber auch, dass zunächst das Wahlrecht im Fokus der Anstrengungen der Frauenrechtler/-innen stand. Für die Zeit nach dem Erhalt des Frauenwahlrechts (1920) bleibt Röwekamps Antwort widersprüchlich. Sie charakterisiert die Strategien zweier führender Organisationen, der $\mathrm{Na}$ tional Women's Party und der National League of Women Voters, als »erfolglos", während sie eine "Explosion an bundesstaatlichen Sorgerechtsreformen« nach feministischen Gleichberechtigungskampagnen feststellt (236).

Deutlicher fällt ihr Urteil in Bezug auf die bürgerliche Frauenrechtsbewegung der Weimarer Republik aus. Die Bewegung hätte »neue recht- liche Ideen ausgedrückt, welche das Dritte Reich überlebten und schließlich das Familienrecht änderten«, was »kaum als ein gescheiterter Fall betrachtet « werden könne (Röwekamp, Gender Difference 234). Die Einführung des Wahlrechts für Frauen und der Verfassung im Jahre 1919, die in den Artikeln 109 und 119 Geschlechtergleichheit festschrieb, bewirkte eine Interessensverschiebung des Bunds deutscher Frauenvereine (BDF) hin zum Familienrecht. Im Bereich des Ehegüterrechts gab es wiederholt Initiativen des BDF zur Ausarbeitung konkreter Reformvorschläge sowie Anträge im Parlament. In der grundlegendsten Form wurde die Trennung des Eigentums in der Ehe ergänzt durch die Zugewinngemeinschaft gefordert. Trotz Unterstützung der Forderungen durch den Deutschen Juristentag und die grundsätzliche Reformabsicht des Justizministeriums kam es vor 1933 nicht mehr zu einem neuen Gesetz. Die Gründe dafür seien allgemeiner Natur gewesen, wie die politische Instabilität der Weimarer Republik, aber auch der Widerstand konservativer und religiöser Interessensverbände und Parteien sowie die Pluralität der Reformwilligen habe dazu beigetragen. Mit den Wurzeln der Haltung konservativer deutscher Kreise hinsichtlich der »Frauenfrage (Frage nach Frauenrechten) « beschäftigt sich Doron Avraham (Gender Difference 197 k. i. O.). Mittels Diskursanalyse erschließt er die für die konservative Ideologie charakteristischen Aspekte zwischen der Revolution von 1848 und der Reichsgründung 1871. Frauen wurde eine spezifische, vermeintlich von Gott vorgesehene und dadurch legitimierte Rolle und Stellung im >organischen` Ganzen der Gesellschaft zugeschrieben, die durch die Unterordnung unter den Ehemann, die Zuordnung in die häusliche Sphäre und die zugeteilte Aufgabe des moralischen Zusammenhalts der Gesellschaft gekennzeichnet war. In dieser "politischen und sozialen Theologie« (207) der Konservativen, die zusätzlich eine zunehmende Desintegration der Gesellschaft wahrnahmen, waren Frauen und Männer zwar vor Gott gleich, eine gesellschaftliche Entwicklung hin zur weltlichen Gleichheit musste jedoch unterbunden werden, um die göttliche Ordnung der Welt nicht zu zersetzen.

Die Frage nach dem Erfolg bzw. Misserfolg der Frauenrechtsbewegungen zieht sich wie ein roter Faden durch nahezu alle Beiträge. Die Wichtigkeit religiöser und politischer Ansichten für die Ausgestaltung der Forderungen der Frauenrechtler/-innen stellt ein wiederkehrendes Motiv dar - >Gleich- 
heit « bedeutete keinesfalls für jede/n dasselbe. Deutlich treten die unterschiedlichen Konjunkturen des Familienrechts hervor: So wurde es bspw. in den USA, England und Deutschland erst schwerpunktmäßig behandelt, nachdem die politischen Rechte erlangt worden waren. Außerdem erhielten Forderungen in verschiedenen familienrechtlichen Bereichen wie Güter-, Sorge- und Scheidungsrecht unterschiedlich viel Zuspruch. Die transnationale Handlungsdimension vieler Akteure/innen der Bewegung, d. h. das Handeln sowohl auf nationaler wie internationaler Ebene, sticht ebenfalls heraus. Diese Ergebnisse sind das Resultat vielfältiger, oftmals miteinander verwobener, methodisch-theoretischer Herangehensweisen der Autoren/-innen, wie Rechts- und Ereignisgeschichte, Biographie und Diskursanalyse; politikwissenschaftlicher, soziologischer und feministischer Konzepte. Leserinnen und Leser können sich einen wissenschaftlich fundierten, kenntnisreichen Überblick zur historischen Problematik >Frauen und Recht « verschaffen, genauso wie sie tiefergehende Informationen und gut strukturierte Analysen zu einzelnen Ländern und/oder Aspekten des Themas finden können.

Dennoch: Mit Blick auf die Methodik bleiben die behandelten Artikel insgesamt konventionell und damit hinter dem Gesamtentwurf der Bände zurück. Komparative Verfahrensweisen sind nur schwach ausgeprägt - die Vergleichsperspektive ergibt sich in der Regel aus den Einleitungen der Herausgeber/-innen und der Nebeneinanderstellung der Artikel. ${ }^{4}$ Gerade hinsichtlich der mul- tiplen Verflechtungen zwischen nationalen und internationalen Handlungs- und Kommunikationsfeldern böten netzwerkanalytische Methoden ein denkbar hohes Potential. Die histoire croisée könnte diesbezüglich ebenfalls Impulse geben, nicht zuletzt weil die Reflektion der Position des Autors / der Autorin integral zu ihrem Repertoire gehört. ${ }^{5}$ Die Haltung der Autoren/-innen schwingt in den Beiträgen jedenfalls häufig mit. Ein Desiderat an der Schnittstelle von Inhalt und methodischer Konzeption ist schließlich der starke Fokus auf die Frauen in der Frauenrechtsbewegung. Im Sinne einer zeitgemäßen Geschlechtergeschichte und ausgewogenen historischen Darstellung wäre auch verstärkt nach dem Beitrag von Männern zu fragen. Inhaltlich wünschenswert für einen umfassenden Überblick wäre zusätzlich eine stärkere schichtspezifische Differenzierung gewesen. Im Mittelpunkt der Beiträge stehen bürgerliche Frauenrechtsforderungen. Inwiefern und auf welche Art und Weise spielten solche Forderungen eine Rolle in anderen sozialen Bewegungen und Kontexten, beispielsweise der Arbeiterbewegung? Ein letzter Wermutstropfen betrifft den Aufbau von Family Law. Wer sich speziell für die Familienrechtsforderungen der deutschen Frauenrechtsbewegung interessiert, wird in diesem Band zu »Western Europe and the United States« nicht fündig und muss den entsprechenden Artikel in Gender Difference zu Rate ziehen. ${ }^{6}$
4 Dieses Urteil trifft nicht auf einige hier nicht angesprochene Beiträge in Gender Difference zu.

5 Vgl. Michael Werner, Bénédicte Zimmermann (2002), Vergleich, Transfer, Verflechtung: Der Ansatz der Histoire croisée und die Herausforderung des Transnationalen, in: Geschichte und Gesellschaft 28,4, 607-636, bes. 622-624, 632 f., 636.
6 Meder und Mecke nennen im Vorwort ein früheres Forschungsprojekt zum deutschen Familienrecht und den zugehörigen Reformdebatten der Frauenrechtsbewegung in Deutschland, auf eine entsprechende Publikation wird allerdings nicht hingewiesen. 